VERSION: JUNE 14, 2016

Preprint typeset using LATEX style emulateapj v. 5/2/11

\title{
INVERSE COMPTON X-RAY EMISSIONS FROM TEV BLAZAR MRK 421 DURING A HISTORICAL LOW-FLUX STATE OBSERVED WITH NUSTAR
}

\author{
JUN KATAOKA ${ }^{1}$ AND ŁUKASZ STAWARZ ${ }^{2}$ \\ Version: June 14, 2016
}

\begin{abstract}
We report on the detection of excess hard X-ray emission from the TeV BL Lac object Mrk 421 during the historical low-flux state of the source in January 2013. NuSTAR observations were conducted four times between MJD 56294 and MJD 56312 with a total exposure of $80.9 \mathrm{ksec}$. The source flux in the $3-40 \mathrm{keV}$ range was nearly constant except for MJD 56307, when the average flux level increased by a factor of three. Throughout the exposure, the X-ray spectra of Mrk 421 were well represented by a steep power-law model with a photon index of $\Gamma \simeq 3.1$, although a significant excess was noted above $20 \mathrm{keV}$ in the MJD 56302 data when the source was in its faintest state. Moreover, Mrk 421 was detected at more than the $4 \sigma$ level in the $40-79 \mathrm{keV}$ count maps for both MJD 56307 and MJD 56302 but not during the remaining two observations. The detected excess hard X-ray emissions connect smoothly with the extrapolation of the high-energy $\gamma$-ray continuum of the blazar constrained by Fermi-LAT during the source quiescence. These findings indicate that, while the overall X-ray spectrum of Mrk 421 is dominated by the highest-energy tail of the synchrotron continuum, the variable excess hard X-ray emission above $20 \mathrm{keV}$ (on the timescale of a week) is related to the inverse Compton emission component. We discuss the resulting constraints on the variability and spectral properties of the low-energy segment of the electron energy distribution in the source.
\end{abstract}

Subject headings: acceleration of particles — radiation mechanisms: non-thermal — galaxies: active - BL Lacertae objects: individual (Mrk 421) — galaxies: jets

\section{INTRODUCTION}

Blazars are a subclass of radio-loud active galactic nuclei (AGN) for which non-thermal jet emissions are relativistically beamed along the line of sight (for a review see, e.g., Begelman et al. 1984; Urry \& Padovani 1995). Thus, pronounced variability on timescales of as short as days and even hours is often observed in various energy bands (e.g., Wagner \& Witzel 1995; Ulrich et al. 1997). The broadband electromagnetic spectra of blazars consist of two characteristic bumps in the $\nu-\nu F_{\nu}$ representation: one extending from radio to optical/X-ray frequencies and the other one peaking around high-energy $\gamma$-ray photon energies. The measured polarization in the radio and optical bands indicates that the low-energy spectral component is due to the synchrotron emissions of ultrarelativistic electrons; the $\gamma$-ray continuum is instead most widely believed to result from inverse Comptonization (IC) of various soft photon fields. The source of the seed photons for the IC process can be either the synchrotron emission of the jet itself ("Synchrotron self-Compton" model, or SSC for short; e.g., Jones et al. 1974; Marscher 1980; Band \& Grindlay 1985), or the thermal emissions of circumnuclear gas and dust (e.g., Dermer \& Schlickeiser 1993; Sikora et al. 1994; Inoue \& Takahara 1996). There is an ongoing debate on the exact localization of the dominant blazar emission zone, with current estimates ranging from hundreds of gravitational radii from central supermassive black holes up to parsec-scale distances, as well as on the dominant particle acceleration processes involved, with different scenarios including mildly relativistic internal shocks (e.g., Böttcher \& Dermer 2010; Mimica \& Aloy 2012; Saito et al.

\footnotetext{
email: kataoka.jun@waseda.jp

${ }^{1}$ Research Institute for Science and Engineering, Waseda University, $3-$ 4-1, Okubo, Shinjuku, Tokyo 169-8555, Japan

${ }^{2}$ Astronomical Observatory of Jagiellonian University, ul. Orla 171, 30-244 Krakow, Poland
}

2015), magnetic turbulence (e.g., Yan et al. 2013; Asano et al. 2014; Zheng et al. 2014; Kakuwa et al. 2015), or even relativistic magnetic reconnection (e.g., Narayan \& Piran 2012; Biteau \& Giebels 2012; Sironi et al. 2015).

Mrk 421 (R.A. $=11 \mathrm{~h} 04 \mathrm{~m} 27.3 \mathrm{~s}$, DEC $=+38 \mathrm{~d} 12 \mathrm{~m} 32$; $z=0.031)$ is a nearby and bright blazar classified as a typical "high-frequency-peaked" BL Lac (HBL) object whose synchrotron and IC emission components extend up to X-ray and very high energy (VHE) $\gamma$-ray photon energies, respectively. It is in fact the first extragalactic source detected in the TeV range (Punch et al. 1992). As such, Mrk 421 is one of the most comprehensively studied HBLs through a number of observation campaigns. In particular, recent multi-wavelength campaigns, including Fermi-LAT , have provided the first ever complete coverage of the $\gamma$-ray continuum of the source in its low-activity/quiescence state (Abdo et al. 2011b). Interestingly, the fractional variability amplitude $F_{\text {var }}$, when plotted as a function of frequency, also reveals a double-peak structure echoing the spectral energy distribution (SED) of Mrk 421: $F_{\text {var }}$ rises significantly from the radio towards the X-ray frequencies, decreases over the Fermi-LAT band, and finally rises again in the $\mathrm{TeV}$ regime (Abdo et al. 2011b; Baloković et al. 2016). This finding seems to imply that, in the framework of the "homogeneous one-zone" emission model, the high-energy tail of the electron energy distribution (synchrotron $\mathrm{X}$-rays and $\mathrm{TeV} \gamma$-rays emitted via the IC process) is highly variable, while the low-energy segment of the electron energy distribution is relatively steady. The alternative interpretation is a stratified (multi-zone) blazar emission model with highly variable high-energy emissions produced in distinct (more compact) emission sites compared with lower-energy emissions, which vary more slowly.

In this context, broadband X-ray observations of Mrk 421 may in principle provide unique clues on the variability and spectral properties of both the low- and the highest-energy 


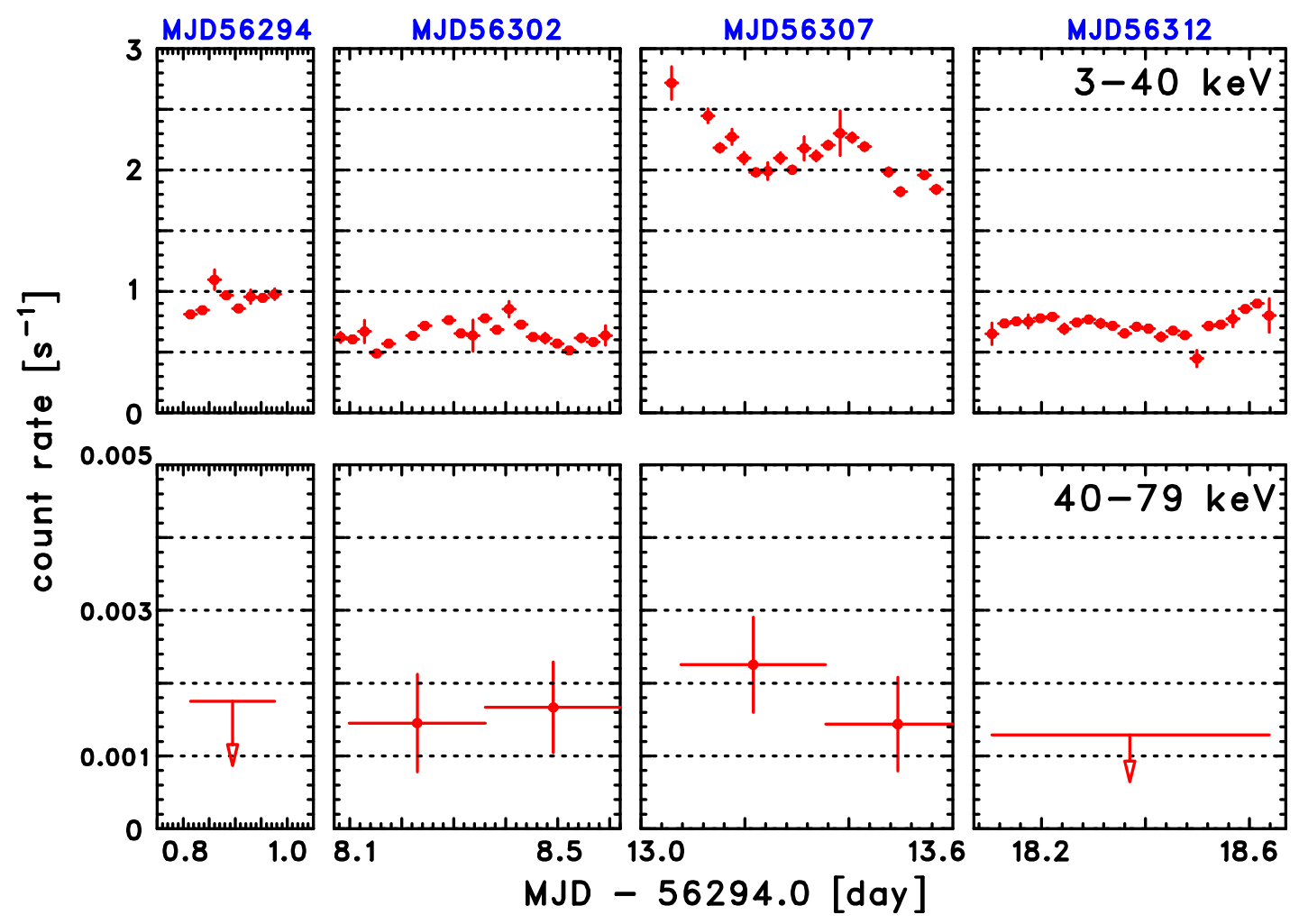

Figure 1. Overall hard X-ray variability of Mrk 421 observed with NuSTAR during the historical low-flux state of the source between MJD 56294 (January 12 , 2013) and MJD 56312 (January 20, 2013). Count rates in the $3-40 \mathrm{keV}$ range (top panels) and $40-79 \mathrm{keV}$ range (bottom panels) are summed from both FPMA and FPMB (after background subtraction) and binned in $2 \mathrm{ks}$ and $20 \mathrm{ks}$ intervals, respectively. For a detection significance of less than $2 \sigma$, upper limits are given for the $95 \%$ confidence level.

segments of the electron energy distribution because the synchrotron continuum of the blazar falls exponentially around photon energies of several/tens of $\mathrm{keV}$ (at least during the low-activity states). This reflects the high-energy cutoff in the underlying electron energy distribution, so the radiative output of the source in the hard X-ray range may be dominated by the low-energy tail of the IC emission component. However, in the case of BL Lacs in general and HBLs in particular, including the X-ray brightest source Mrk 421, X-ray data above $10 \mathrm{keV}$ are deficient and available typically only for isolated flaring periods (see Ushio et al. 2009, 2010; Abdo et al. 2011b). This is because previous hard X-ray observations relied on "non-imaging" detectors for which the sensitivity was insufficient to detect the source in its low-activity states. The Nuclear Spectroscopic Telescope Array mission (NuSTAR ; Harrison et al. 2013) is the first focusing high-energy X-ray telescope in orbit and operates in the band from 3 to $79 \mathrm{keV}$. Thanks to its imaging capability, NUSTAR probes the hard Xray sky with a more than 100 -fold improvement in sensitivity.

Paliya et al. (2015) and Sinha et al. (2015) presented the NUSTAR observations of Mrk 421 during flaring epochs. In this paper, we revisit the archival Mrk 421 data provided by NUSTAR in January 2013 and discussed previously by Baloković et al. (2016) when the source was in a historical low-activity state corresponding to an extrapolated $2-10 \mathrm{keV}$ energy flux of $\simeq 4 \times 10^{-11} \mathrm{erg} \mathrm{cm}^{-2} \mathrm{~s}^{-1}$.

\section{OBSERVATIONS AND RESULTS}

\subsection{Observation and Data Reduction}

Mrk 421 was selected as a representative of the HBL class of blazars and thus has been observed frequently with $\mathrm{NuS}$ -
TAR since January 2013, partly in the framework of extensive multi-frequency campaigns. The total exposure time over 88 orbits amounts to more than $250 \mathrm{ks}$, including two calibrating observations conducted in July 2012 (Baloković et al. (2016). A typical NuSTAR observation spans $10 \mathrm{hrs;}$ hence, the actual exposure for each observation, after only "good time intervals" are selected to eliminate the passage of the South Atlantic Anomaly and orbital modulation of visibility, ranges from $10 \mathrm{ks}$ to $25 \mathrm{ks}$. All observations were conducted with two co-aligned independent telescopes called Focal Plane Module A and B (FPMA and FPMB). Throughout the observations, variations in the count rate of more than two orders of magnitude were observed. It has been argued that the synchrotron emissions of the blazar account for all the flux detected up to $79 \mathrm{keV}$ during the flaring states (Paliya et al. 2015; Sinha et al. 2015). Here, we focus on the historical low-flux state of Mrk 421, which was witnessed by NuSTAR in January 2013 (see Table 1 for the logs of the analyzed observations).

The archival NUSTAR data were downloaded from NASA's HEASARC interface 3 and reduced by using the NuSTARDAS software, which is included as part of the HEAsoft package version 6.17. For the temporal and spectral studies presented in the following sections, the X-ray events were extracted from a circular region with a radius of $30^{\prime \prime}$ and centred on the source position, whereas the background was accumulated in an annulus with inner and outer radii of $30^{\prime \prime}$ and $70^{\prime \prime}$, respectively. We carefully checked that other choices of source and background regions did not affect the analysis

\footnotetext{
${ }^{3}$ http://heasarc.gsfc.nasa.gov/docs/archive.html
} 

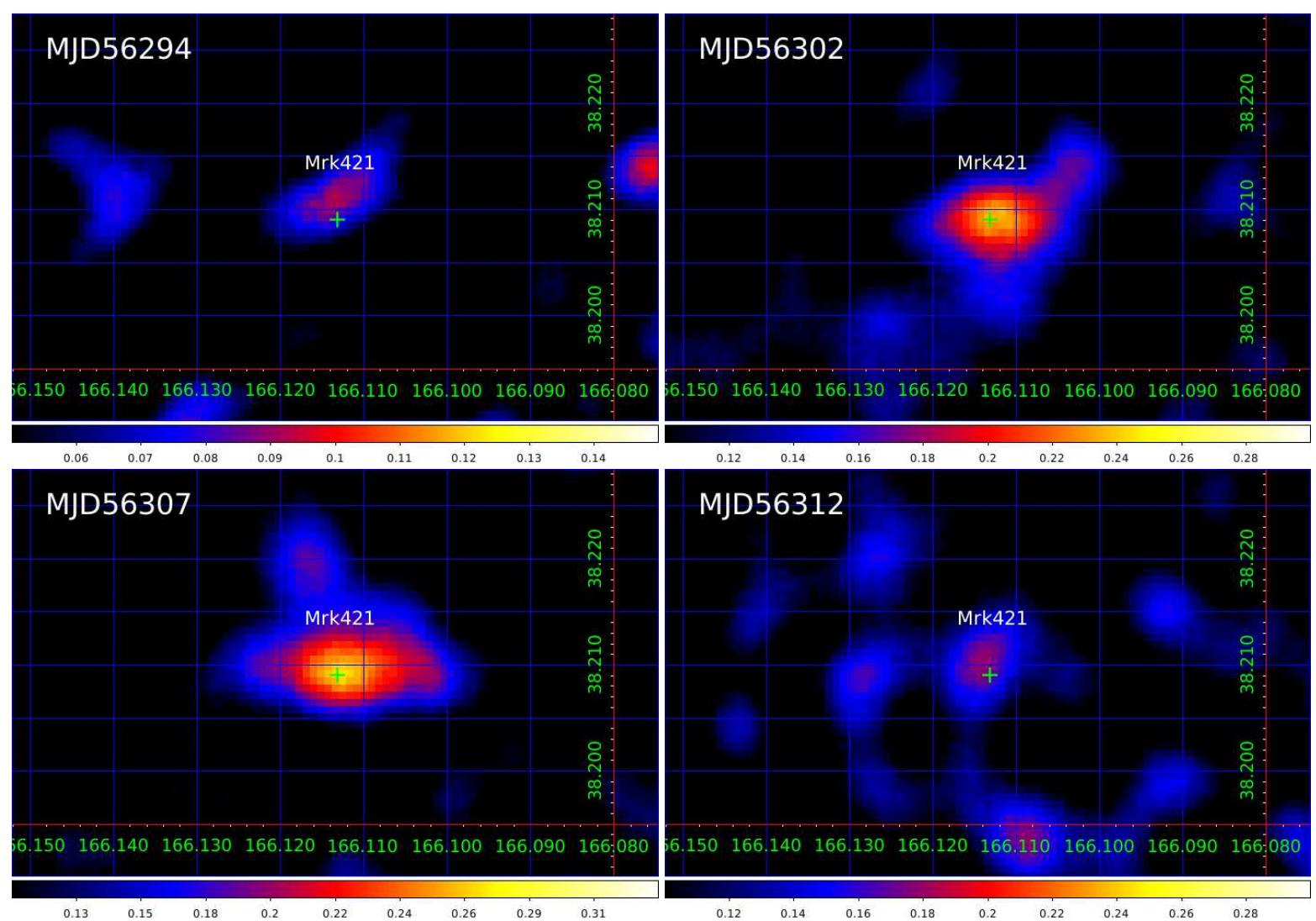

Figure 2. Temporal variations in the NuSTAR images of Mrk 421 in the $40-79 \mathrm{keV}$ energy range. The images denotes the relative excess of photon counts (arbitrary units indicated in the color bar corrected for difference in exposures; see Table 1 s smoothed with a Gaussian kernel of 19.6" . Photon counts from FPMA and FPMB are summed. Note the source was significantly detected at more than the $4 \sigma$ level only in MJD56302 and MJD56307, which consistent with the source light curve given in Figure 1

results presented in the next sections within uncertainties of $1 \sigma$. The response files were generated by using the standard nupipel ine and nuproduct s scripts. Version 20151008 of the CALDB files were used in this study. Even in the historical low-flux state of the target, the extracted X-ray count rates were well above the background level up to $40 \mathrm{keV}$ and almost equal to the background level above $40 \mathrm{keV}$. With good characterization of the NUSTAR background, which left the uncertainty at a 1\% level (Wik et al. 2014), the gathered Mrk 421 data could therefore be used for spectral modeling up to the high-energy end of the NUSTAR band at $79 \mathrm{keV}$.

\subsection{Analysis Results}

Figure 1 shows the count rate variations during the $N u S$ TAR observations between January 2 (MJD 56294) and January 20 (MJD 56312). The X-ray counts from both FPMA and FPMB detectors are summed, and the background is subtracted. The light curves are shown separately for the two different energy bands: $3-40 \mathrm{keV}$ (top panels) with $2 \mathrm{ks}$ binning and $40-79 \mathrm{keV}$ (bottom panels) with $20 \mathrm{ks}$ binning. The light curves indicate that the $3-40 \mathrm{keV}$ source flux was nearly constant except for MJD 56307 when a pronounced variability was observed and the average flux increased by a factor of three. In addition, note that the source was detected at more than the $3 \sigma$ level in the $40-79 \mathrm{keV}$ band not only in MJD 56307 but also in MJD 56302, when an average $3-40 \mathrm{keV}$ count rate was lowest. For the remaining two observations, we provide the corresponding $95 \%$ confidence level upper limits.
Figure 2 compares the temporal variations in the NuSTAR X-ray images of Mrk 421 in the $40-79 \mathrm{keV}$ energy range. These were reconstructed by using the sum of the FPMA and FPMB data in MJD 56294, 56302, 56307 and 56312, respectively. The images were smoothed by a Gaussian kernel of $19.6^{\prime \prime}$ and color-coded; the given color bar indicates a relative excess of photon counts in arbitrary units for which the maximum value is corrected for different exposure times of each observation (see Table 1). Note that the target was clearly detected at more than the $4 \sigma$ level only in MJD 56302 and 56307 , which is consistent with the source light curve given in Figure 1. A slightly higher significance for the images $(>4 \sigma)$ compared to the light curves $(>3 \sigma)$ is because source counts were extracted from a circular region with a radius of $30^{\prime \prime}$ for Figure 1, whereas all of the detected photons were used for Figure 2 against the point spread function of NuSTAR . A positive detection of the source above $40 \mathrm{keV}$ during the extremely low-flux state in MJD 56302 but not during similarly low-flux states in MJD 56294 and 56312 indicates that the excess hard X-ray emission is variable on the time scale of a week.

For the spectral fitting, we binned the source light curve to a minimum of 40 counts per bin to enable the $\chi^{2}$ minimization statistics. Table 1 lists the resulting fitting parameters for all of the analyzed observations, for which errors are quoted at the $1 \sigma$ confidence level. The Galactic absorption column density toward Mrk 421 was taken to be $N_{\mathrm{H}}=1.5 \times 10^{20} \mathrm{~cm}^{-2}$ (Elvis et al. 1989). Figure 3 shows the FPMA (black symbols) and FPMB (red symbols) spectra during MJD 56302 
Table 1

Summary of NUSTAR observations and analysis of Mrk 421

\begin{tabular}{|c|c|c|c|c|c|c|c|}
\hline $\begin{array}{l}\text { Start Time } \\
\text { (MJD) }\end{array}$ & $\begin{array}{c}\text { Exposure } \\
(\mathrm{ksec})\end{array}$ & model & $\Gamma_{1}^{d}$ & $\begin{array}{l}E_{\mathrm{brk}}^{e} \\
(\mathrm{keV})\end{array}$ & $\Gamma_{2}^{f}$ & $\begin{array}{l}3-79 \mathrm{keV} \text { energy flux } \\
\left(10^{-12} \mathrm{erg} \mathrm{cm}^{-2} \mathrm{~s}^{-1}\right)\end{array}$ & $\chi^{2} / \mathrm{dof}$ \\
\hline \multirow[t]{3}{*}{56294.78} & 9.2 & $\mathrm{PL}^{a}$ & $3.10 \pm 0.03$ & - & - & $44.4 \pm 0.6$ & $170.8 / 161$ \\
\hline & & $\mathrm{BKN}^{-\mathrm{PL}^{b}}$ & $3.25 \pm 0.05$ & $7.23 \pm 0.66$ & $2.87_{-0.07}^{+0.06}$ & $46.4 \pm 4.2$ & $155.1 / 159$ \\
\hline & & $\mathrm{PL}+\mathrm{PL}^{c}$ & $3.32 \pm 0.07$ & - & $1.7^{f}$ & $48.4 \pm 0.9$ & $158.1 / 160$ \\
\hline \multirow[t]{3}{*}{56302.05} & 22.6 & $\mathrm{PL}$ & $3.06 \pm 0.02$ & - & - & $31.0 \pm 0.3$ & $262.3 / 249$ \\
\hline & & BKN-PL & $3.08 \pm 0.02$ & $17.8_{-1.6}^{+2.1}$ & $2.08_{-0.28}^{+0.26}$ & $34.0 \pm 1.3$ & $246.3 / 247$ \\
\hline & & $\mathrm{PL}+\mathrm{PL}$ & $3.11_{-0.03}^{+0.04}$ & - & $0.17_{-0.79}^{+0.71}$ & $35.3 \pm 0.5$ & $247.9 / 247$ \\
\hline \multirow[t]{2}{*}{56307.04} & 24.2 & $\overline{P L}$ & $3.03 \pm 0.01$ & - & - & $107.6 \pm 0.5$ & $467.4 / 456$ \\
\hline & & BKN-PL & $2.96 \pm 0.02$ & $7.83_{-0.5}^{+0.6}$ & $3.19_{-0.03}^{+0.04}$ & $105.1 \pm 3.3$ & $429.6 / 454$ \\
\hline \multirow[t]{2}{*}{56312.10} & 25.0 & PL & $3.07 \pm 0.02$ & - & - & $36.4 \pm 0.3$ & $295.3 / 290$ \\
\hline & & BKN-PL & $3.07 \pm 0.02$ & $28.8_{-5.2}^{+9.2}$ & $1.78_{-1.78}^{+0.81}$ & $38.2 \pm 1.2$ & $292.5 / 288$ \\
\hline
\end{tabular}

Note. $-{ }^{a}$ : The power-law model, WABS*PEGP, where the absorption column density was fixed to the Galactic value in the direction of the source, $N_{\mathrm{H}}=1.5 \times 10^{20} \mathrm{~cm}^{-2}$.

${ }^{b}$ : The broken power-law model, WABS*BKENPOWER, where the absorption column density was fixed to the Galactic value.

${ }^{c}$ : The double power-law model, WABS*(PEGP+PEGP), where the absorption column density was fixed to the Galactic value.

$d$ : The power-law index for the PL model, the low-energy power-law index for the BKN-PL model, or finally the power-law index for the first PL components in the PL+PL model.

${ }^{e}$ : The break energy for the BKN-PL model in $\mathrm{keV}$.

${ }^{f}$ : The high-energy power-law index for the BKN-PL model, or the power-law index for the second PL components for the PL+PL model.

plotted against the best-fit power-law (PL), broken power-law (BKN-PL) in which high-energy photon index is harder than that in the low-energy part, and double power-law (PL+PL) models within the energy range of $3-79 \mathrm{keV}$. The residuals in the figure corresponding to the PL fit (best-fit photon in$\operatorname{dex} \Gamma=3.10 \pm 0.03$ with $\chi^{2} /$ dof of $262.3 / 249$ ) indicate that the source spectrum exhibits significant hard excess emissions above $20 \mathrm{keV}$. The fit was significantly improved in the cases of both the BKN-PL and PL+PL models ( $\chi^{2} /$ dof of $246.3 / 247$ and $247.9 / 247$ dof, respectively). Thus, the improvement in the $\chi^{2}$ statistic as measured with the $F$ static value of 8.0 was significant at more than the $99.9 \%$ level using the $F$-test. The spectral hardening was statistically less significant in the MJD 56294 and 56312 data (see Table1).

\section{DISCUSSION AND CONCLUSION}

In the previous sections we presented an analysis of the archival NuSTAR observations of Mrk 421 in January 2013 when the overall X-ray flux was particularly low. In MJD 56032, the $2-10 \mathrm{keV}$ energy flux estimated from the extrapolation of the PL fit (photon index $\Gamma \simeq 3.1$ ) was $(4.02 \pm 0.05) \times 10^{-11} \mathrm{erg} \mathrm{cm}^{-2} \mathrm{~s}^{-1}$, which is the lowest ever reported in the literature for the source. The detection of Mrk 421 above $40 \mathrm{keV}$ in such a low flux state manifested in a concave broadband X-ray spectrum of the target with excess hard X-ray emissions dominant at photon energies above $20 \mathrm{keV}$ (Table 1 and Figure 3). However, this excess was not found in the MJD 56294 or 56312 data when the source was in a similarly low-flux state (Figures 1 and 2), which implies a variability of the hard X-ray continuum on the timescale of a week.

To investigate the physical origin of the detected excess emission, we compiled the broad-band X-ray $(N u S T A R$; this work) and the high-energy $\gamma$-ray (Fermi-LAT; Abdo et al. 2011b) data in a $\nu-\nu F_{\nu}$ representation, as shown in Figure 4. Bow-ties plotted in black denote the Fermi-LAT data taken during different multi-frequency campaigns; the average source spectrum during the period of a source quiescence (from 2008 August 5 to 2010 February 20) is shown as a magenta bow-tie. The dashed magenta lines mark the extrap- olation of the best-fit power-law model applied to the average Fermi-LAT data $\left(\gamma\right.$-ray photon index of $\Gamma_{\gamma} \sim 1.78 \pm$ 0.02 ; Abdo et al. 2011b), with $1 \sigma$ uncertainty. The excess hard X-ray emissions detected in the analyzed NuSTAR data agreed well with the extrapolation of the average Fermi-LAT spectrum. This indicates that the quiescence continuum of Mrk 421, which extends from photon energies of tens of $\mathrm{keV}$ up to tens of $\mathrm{GeV}$, corresponds to a single IC emission component and is well-described by a power-law model with a photon index of $\simeq 1$. 8 .

Interestingly, similar spectral upturns related to the synchrotron/IC crossover have been detected below $10 \mathrm{keV}$ in several other blazars classified as "low-frequency-peaked" BL Lacs (LBLs; see, e.g., Tagliaferri et al. 2000; Tanihata et al. 2003; Wierzcholska \& Wagner 2016) but never in HBLs either below or above $10 \mathrm{keV}$. Further novelty here is that the high-energy excess feature found in the NuSTAR data for Mrk 421 is variable on the time scale of a week; no evidence for such variability in the low-energy segment of the IC emission continuum has been reported for other BL Lacs in the literature.

An alternative explanation for the observed hard X-ray excess of the target is a spectral pile-up in the electron distribution $N_{e}^{\prime}\left(\gamma_{e}\right)$, where $\gamma_{e}$ is the electron Lorentz factor, forming temporarily at the highest energies 4 This feature may appear due to either (i) a continuous (stochastic) acceleration of electrons limited by radiative losses (e.g., Stawarz \& Petrosian 2008), or (ii) the reduction of the IC cross section in the KleinNishina regime (e.g., Moderski et al. 2005). In the case of (i), the pile-up bump appears at the maximum electron energies for which the acceleration timescale equals the radiative loss timescale at the limit for the perfect confinement of electrons

\footnotetext{
${ }^{4}$ Here we do not consider a possibility that the observed hard X-ray excess is related to the bulk Comptonization of the accretion disk emission by cold electrons within the innermost parts of Mrk 421 jet, since the corresponding bulk-Compton spectral features have been predicted for (and possibly even observed in) luminous blazars of the "flat-spectrum-radio-quasar" type (see, e.g., Sikora et al. 1997; Kataoka et al. 2008, and references therein), and not for BL Lac objects characterized by low accretion rates and hence radiatively inefficient accretion disks.
} 


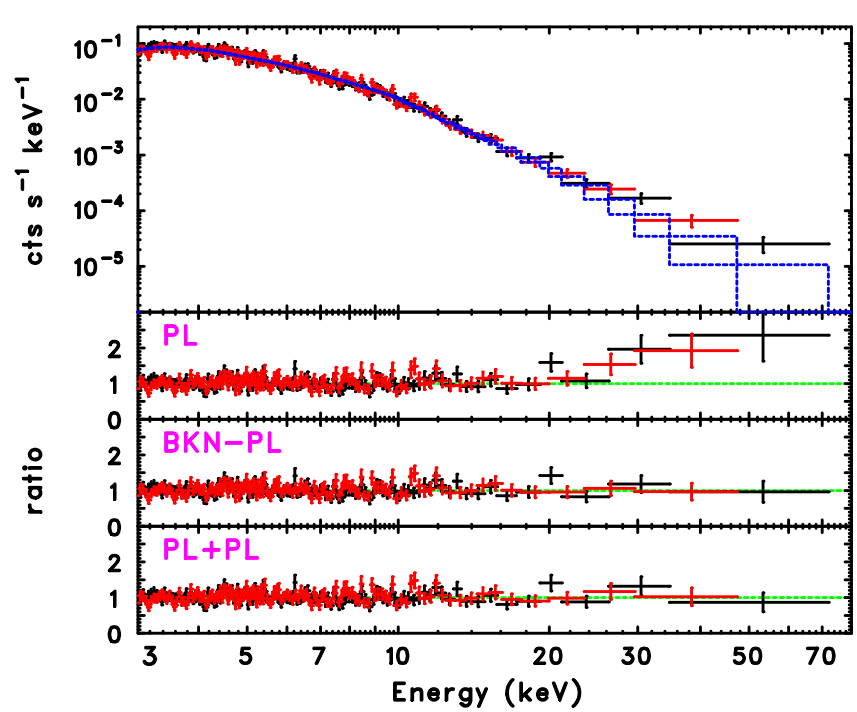

Figure 3. NUSTAR spectrum of Mrk 421 from the MJD 56302 data. The upper panel presents the FPMA (black) and FPMB (red) data plotted against an absorbed power-law model with the photon index $\Gamma \simeq 3.1$ and the Galactic column density of $N_{\mathrm{H}}=1.5 \times 10^{20} \mathrm{~cm}^{-2}$, fitted over the $3-79 \mathrm{keV}$ band. The bottom panels show the data/model ratio residuals for the power-law fit (PL), broken power-law fit (BKN-PL), and double power-law fit (PL+PL). Deviations above $20 \mathrm{keV}$ are clearly seen in the PL fitting, as detailed in the text.

within the emission zone (i.e., no particle escape). However, the power-law tail at lower electron energies has to be relatively flat in such a scenario, $s \equiv-d \ln N_{e}^{\prime}\left(\gamma_{e}\right) / d \ln \gamma_{e}=$ $0-1$, which disagrees with the observation in Mrk 421. Similarly, in the case of (ii), the necessary condition for the formation of a pronounced spectral hardening in the electron energy distribution is the dominance of IC cooling over the synchrotron one and a relatively narrow (within the frequency range) seed photon distribution. These requirements contradict the conditions expected for the Mrk 421 jet in its quiescence (for which the synchrotron losses dominate the IC ones and the soft photon distribution for the IC scattering is provided by the broadband synchrotron emission of the jet itself). Therefore, while also considering the extrapolation of the Fermi-LAT spectrum of the source to the X-ray regime, we argue that the hard X-ray excess found in the NuSTAR data indeed represents the low-energy tail of the IC (SSC) component.

From the observed SSC photon energy of $h \nu_{\mathrm{SSC}} \simeq 20 \mathrm{keV}$ and a steep broadband power-law electron energy distribution, the corresponding minimum electron Lorentz factor is roughly

$$
\gamma_{e, \min } \sim 10^{3}\left(\frac{B^{\prime}}{0.1 \mathrm{G}}\right)^{-1 / 4}\left(\frac{\delta}{10}\right)^{-1 / 4} .
$$

The radiative cooling timescale for such low-energy electrons dominated by the synchrotron process is then

$$
\tau_{\text {cool }}\left(\gamma_{e, \min }\right) \sim 10^{7}\left(\frac{B^{\prime}}{0.1 \mathrm{G}}\right)^{-7 / 4}\left(\frac{\delta}{10}\right)^{-3 / 4} \mathrm{~s},
$$

which is much longer than a week for the comoving magnetic field intensity $B^{\prime} \lesssim 0.1 \mathrm{G}$ emerging from the one-zone SSC model applied to the quiescence SED of Mrk 421 (Abdo et al. 2011b). Hence, the variability of the low-energy electrons implied by the NUSTAR observations analyzed in this paper have

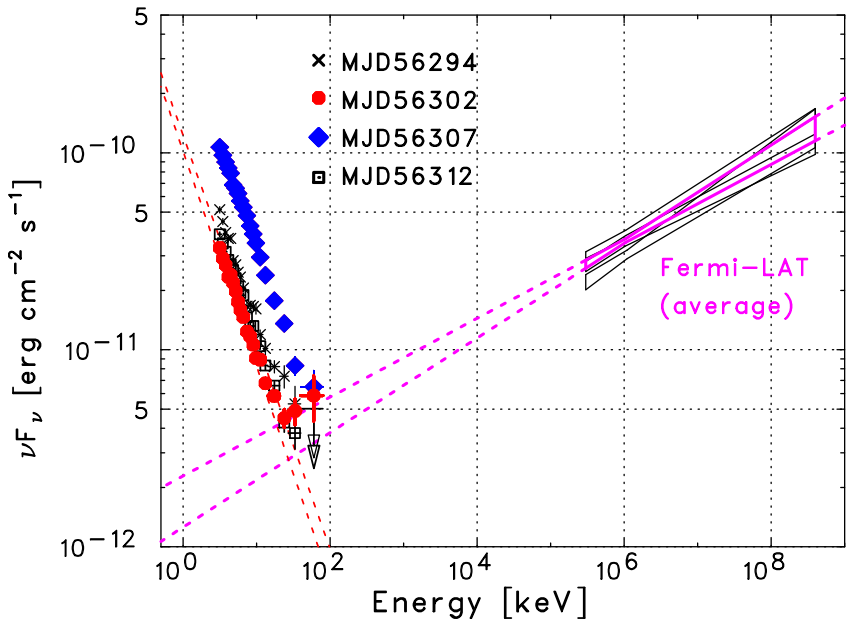

Figure 4. Broadband $\mathrm{X}$-ray (NUSTAR ; this paper) to high-energy $\gamma$-ray (Fermi-LAT; Abdo et al. 2011b) spectra of Mrk 421. Bow-ties plotted in black denote the Fermi-LAT data taken in different multi-frequency campaigns; the average source spectrum during the period of a source quiescence (from 2008 August 5 to 2010 February 20) is shown as a magenta bow-tie. Dashed magenta lines mark the extrapolation of the best-fit power-law model to the average Fermi-LAT data ( $\gamma$-ray photon index of $1.78 \pm 0.02$ ).

to be related to dynamical changes within the blazar emission zone for which the shortest timescale is given by the light crossing time $R / c$. Interestingly, this would agree with the emission region size assumed in the SED model of Abdo et al. (2011b). In particular, one has

$$
R \simeq 0.06\left(\frac{t_{\mathrm{var}}}{1 \text { week }}\right)\left(\frac{\delta}{10}\right) \mathrm{pc},
$$

which implies the distance of the emission zone from the active nucleus $r \sim \Gamma_{j} R \sim 0.6 \mathrm{pc}$ for the anticipated conical jet geometry with the opening angle $\sim 1 / \Gamma_{j}$ and the jet bulk Lorentz factor $\Gamma_{j} \sim \delta \sim 10$. This is also in accord with the detailed analysis of the overall variability of Mrk 421 at $\mathrm{X}$-ray frequencies, which implies that the power in the intraday flickering of the source is small (Kataoka et al. 2001; Isobe et al. 2015).

In addition, the results of our NUSTAR data analysis revealed that the electron energy distribution in Mrk 421 during the source quiescence is well-represented by a relatively steep power law with the energy index of $s \simeq 2 \Gamma_{\gamma}-1 \sim 2.6$, which extends from electron energies of at least $\gamma_{e} \sim 10^{3}$ up to $\gamma_{e} \sim 10^{6}$ (in the jet rest frame). This is an important finding because in such a case the bulk of the jet kinetic energy is carried by those low-energy electrons (assuming no significant proton content). The exact value of the electron spectral index is also important based on the most recent results for the kinetic simulations of relativistic magnetic reconnection process. In other words, the power-law energy spectra formed within the reconnection sites are steep $(s>2$ only if the jet magnetization is low (Sironi \& Spitkovsky 2014; Guo et al. 2015). Again, low magnetization of the emission region would be in accord with the model parameters emerging from a simple one-zone SSC models (Abdo et al. 2011a b). However, note that this conclusion is with regard to only the slowly variable/quiescence emission component because the production of rapid high-amplitude flares in blazar sources may proceed under very different conditions, and may involve very different electron spectra. 
Ł.S. was supported by Polish NSC grant DEC2012/04/A/ST9/00083.

\section{REFERENCES}

Abdo, A. A., Ackermann, M., Ajello, M., et al. 2011a, ApJ, 727, 129 Abdo, A. A., Ackermann, M., Ajello, M., et al. 2011b, ApJ, 736, 131 Asano, K., Takahara, F., Kusunose, M., Toma, K., \& Kakuwa, J. 2014, ApJ, 780,64

Baloković, M., Paneque, D., Madejski, G., et al. 2016, ApJ, 819, 156

Band, D. L., \& Grindlay, J. E. 1985, ApJ, 298, 128

Begelman, M. C., Blandford, R. D., \& Rees, M. J. 1984, Reviews of Modern Physics, 56, 255

Biteau, J., \& Giebels, B. 2012, A\&A, 548, A123

Böttcher, M., \& Dermer, C. D. 2010, ApJ, 711, 445

Dermer, C. D., \& Schlickeiser, R. 1993, ApJ, 416, 458

Elvis, M., Wilkes, B. J., \& Lockman, F. J. 1989, AJ, 97, 777

Guo, F., Liu, Y.-H., Daughton, W., \& Li, H. 2015, ApJ, 806, 167

Harrison, F. A., Craig, W. W., Christensen, F. E., et al. 2013, ApJ, 770, 103

Inoue, S., \& Takahara, F. 1996, ApJ, 463, 555

Isobe, N., Sato, R., Ueda, Y., et al. 2015, ApJ, 798, 27

Jones, T. W., O’Dell, S. L., \& Stein, W. A. 1974, ApJ, 188, 353

Kakuwa, J., Toma, K., Asano, K., Kusunose, M., \& Takahara, F. 2015, MNRAS, 449, 551

Kataoka, J., Takahashi, T., Wagner, S. J., et al. 2001, ApJ, 560, 659

Kataoka, J., Madejski, G., Sikora, M., et al. 2008, ApJ, 672, 787-799

Marscher, A. P. 1980, ApJ, 235, 386
Mimica, P., \& Aloy, M. A. 2012, MNRAS, 421, 2635

Moderski, R., Sikora, M., Coppi, P. S., \& Aharonian, F. 2005, MNRAS, 363, 954

Narayan, R., \& Piran, T. 2012, MNRAS, 420, 604

Paliya, V. S., Böttcher, M., Diltz, C., et al. 2015, ApJ, 811, 143

Punch, M., Akerlof, C. W., Cawley, M. F., et al. 1992, Nature, 358, 477

Saito, S., Stawarz, Ł., Tanaka, Y. T., et al. 2015, ApJ, 809, 171

Sikora, M., Begelman, M. C., \& Rees, M. J. 1994, ApJ, 421, 153

Sikora, M., Madejski, G., Moderski, R., \& Poutanen, J. 1997, ApJ, 484, 108

Sinha, A., Shukla, A., Misra, R., et al. 2015, A\&A, 580, A100

Sironi, L., \& Spitkovsky, A. 2014, ApJ, 783, L21

Sironi, L., Petropoulou, M., \& Giannios, D. 2015, MNRAS, 450, 183

Stawarz, Ł., \& Petrosian, V. 2008, ApJ, 681, 1725-1744

Tagliaferri, G., Ghisellini, G., Giommi, P., et al. 2000, A\&A, 354, 431

Tanihata, C., Takahashi, T., Kataoka, J., \& Madejski, G. M. 2003, ApJ, 584, 153

Ulrich, M.-H., Maraschi, L., \& Urry, C. M. 1997, ARA\&A, 35, 445

Ushio, M., Tanaka, T., Madejski, G., et al. 2009, ApJ, 699, 1964

Ushio, M., Stawarz, Ł., Takahashi, T., et al. 2010, ApJ, 724, 1509

Urry, C. M., \& Padovani, P. 1995, PASP, 107, 803

Wagner S. J., Witzel A. 1995, ARA\&A, 33, 163

Wierzcholska, A., \& Wagner, S. J. 2016, MNRAS, 458, 56

Wik, D. R., Hornstrup, A., Molendi, S., et al. 2014, ApJ, 792, 48

Yan, D., Zhang, L., Yuan, Q., Fan, Z., \& Zeng, H. 2013, ApJ, 765, 122

Zheng, Y. G., Kang, S. J., \& Li, J. 2014, MNRAS, 442, 3166 